\title{
Using Pegylated Interferon alfa-2b and Ribavirin to Treat Chronic Hepatitis Patients Infected with Hepatitis C Virus Genotype 1: Are Nonresponders and Relapsers Different Populations?
}

\author{
Giovanni Faria Silva1, Rodrigo José Polônioํ, Maria Inês Moura Campos Pardini², Silvia Maria Corvino ${ }^{2}$, Rita Maria Saccomano \\ Henriques $^{2}$, Mari Nilce Peres ${ }^{1}$, Liciana Vaz Arruda Silveira and Kunie Iabuki Rabello Coelho ${ }^{3}$ \\ ${ }^{1}$ Department of Internal Medicine; ${ }^{2}$ Laboratory of Virology, Hemocenter of Botucatu; ${ }^{3}$ Department of Pathology; Botucatu, SP, Brazil
}

\begin{abstract}
The combination of pegylated interferon (PEG-INF) and ribavirin is currently the best treatment for chronic hepatitis C, providing a sustained virological response (SVR) in 54\%-63\% of patients. In patients infected with hepatitis C virus (HCV) genotype 1, the SVR rate is $42 \%-52 \%$. To evaluate the treatment efficacy of this drug combination, we conducted an open, prospective study of 58 consecutive treatment-naïve patients infected with HCV genotype 1 and treated at a university hospital, comparing those presenting an SVR (SVRs), nonresponders (NRs), and relapsers (RELs). Among the intent-to-treat patients, an end-of-treatment virological response was achieved in $69 \%$ of the sample as a whole and in $52 \%$ of the SVRs. We found that being an SVR was significantly associated with mild fibrosis $(p=0.04)$ and with undetectable HCV RNA at weeks 12 and 24 of treatment $(p<0.0001)$. Comparing the SVR and REL groups, we observed that being older than 40 was significantly associated with being a REL $(p=0.04)$. Being an NR was found to be associated with severe fibrosis and moderate inflammatory infiltrates (portal or periportal). In the polytomous logistic regression, no independent factors were associated with the REL group when compared with the SVR group. We conclude that RELs and NRs differ in comparison with SVRs. The RELs accounted for $17 \%$ of the sample. The HCV RNA test results at weeks 12 and 24 of treatment, although independent predictors of non-response (OR: 4.8 and 8.2, respectively), did not differ between SVRs and RELs.
\end{abstract}

Key-Words: Hepatitis $\mathrm{C}$, pegylated interferon alfa $2 \mathrm{~b}$, genotype 1, relapsers.

Approximately 200 million people worldwide are infected with hepatitis $\mathrm{C}$ virus (HCV). It is one of the main causes of cirrhosis worldwide and is the leading indication for hepatic transplantation in the USA $[1,2]$.

Currently, the best treatment for chronic HCV infection is the combination of pegylated interferon and ribavirin. This combination results in cure, which is defined as a sustained virological response (SVR, HCV RNA undetectable at week 24 after end of treatment), in 54\%-63\% of patients [3-5]. Patients with HCV genotype 1 (the most common strain) constitute the most difficult subgroup to treat, presenting SVR rates ranging from $42 \%$ to $52 \%$ [3-5]. Presenting an SVR has been associated with arresting the progression, or even leading to the regression of hepatic disease [3,6-8]. Therefore, achieving an SVR is the main objective of the treatment.

The factors that are known to be predictive of an SVR are as follows: being infected with a genotype other than genotype 1 [3-5]; having a low viral load [3,5]; presenting no cirrhosis or foci of fibrosis [3]; being 40 years of age or less $[3,4]$; and weighing less than $75 \mathrm{~kg}$ [4]. Viral load clearance (absence of or $2-\log$ reduction in the viral load in relation to the pretreatment value) at week 12 of treatment is also a strong predictor of SVR [4].

Patients who do not present an SVR include nonresponders (HCV RNA detectable at the end of treatment) and relapsers (HCV RNA undetectable at end of treatment

Received on 6 July 2007; revised 27 October 2007.

Address for correspondence: Dr. Giovanni Faria Silva. Department of Internal Medicine - Faculdade de Medicina de Botucatu - UNESP. Zip code: 18618-000 - Botucatu, SP, Brazil. E-mail: giovanni@fmb.unesp.br.

The Brazilian Journal of Infectious Diseases 2007;11(6):554-560. (C) 2007 by The Brazilian Journal of Infectious Diseases and Contexto Publishing. All rights reserved. but again detectable within 6 months after the end of treatment).

Studies evaluating the pegylated interferon-ribavirin combination as a treatment for HCV infection have shown relapse rates ranging from $13 \%$ to $18 \%[3,4]$. However, those studies involved patients infected with all of the HCV genotypes. Studies attempting to identify SVRs have not discriminated between nonresponders and relapsers. Therefore, we can not know whether the factors predictive of not presenting an SVR would be the same for these two patient groups. If we could predict which patients initially classified as responders would later be classified as nonresponders (i.e., relapsers), we could take measures (perhaps prolonging the treatment) in order to achieve an SVR in such patients.

In view of this, we evaluated the efficacy of treatment with pegylated interferon alfa $2 \mathrm{~b}$ and ribavirin in patients presenting chronic infection with HCV genotype 1. We also attempted to characterize the possible differences between nonresponders and relapsers. In addition, we sought to identify factors associated with the transition from responder to relapser.

\section{Material and Methods \\ Patients and Study Design}

In this open, prospective study, we recruited 58 of 84 consecutive patients with chronic HCV infection and seeking treatment at the Gastroenterology outpatient clinic of the Paulista State University School of Medicine at Botucatu between January $1^{\text {st }}$ and October 10, 2004. The selected patients received pegylated interferon alfa $2 \mathrm{~b}$ (Schering Corp., Kenilworth, NJ, USA) combined with ribavirin. In the present study, patients were treated and monitored in an academic environment at a community health care facility. Therefore, the data collected can be considered 'real-world' data. 
Inclusion criteria were as follows: HCV RNA detectable by polymerase chain reaction (PCR) in serum; presenting infection with $\mathrm{HCV}$ genotype 1; liver biopsy consistent with chronic hepatitis; treatment-naïve patients; and being treated with the combination of pegylated interferon alfa $2 b$ and ribavirin.

We also employed the following exclusion criteria: presenting co-infection with hepatitis B or human immunodeficiency virus; having decompensated cirrhosis, being under treatment with pegylated interferon alfa $2 \mathrm{a}$; presenting hematological values that are below the normal minimums (platelet counts of $50,000 / \mathrm{mm}^{3}$ and neutrophil counts of $1,500 / \mathrm{mm}^{3}$, as well as hemoglobin of $12 \mathrm{mg} / \mathrm{dL}$ for women and $13 \mathrm{mg} / \mathrm{dL}$ for men).

Eligible patients received weekly subcutaneous injections of pegylated interferon alfa $2 \mathrm{~b}(1.5 \mu \mathrm{g} / \mathrm{Kg})$ and daily doses of oral ribavirin $(1,000 \mathrm{mg}$ for those weighing $<75 \mathrm{Kg}$ and 1,250 $\mathrm{mg}$ for those weighing $>75 \mathrm{Kg}$ ). Once a week, the patients were weighed, after which the pegylated interferon alfa $2 b$ was administered, and the patients were evaluated by a multidisciplinary team composed of physicians, nurses, psychologists and nutritionists.

Over the course of the treatment, patients were submitted to hematological and biochemical tests on a weekly or monthly basis, depending on patient clinical status. Testing for HCV RNA by PCR was carried out at weeks 12, 24, and 48 of treatment, as well as at post-treatment weeks 12 and 24.

Treatment efficacy was defined as the presence of an SVR (HCV RNA undetectable at post-treatment week 24). The efficacy analysis was carried out in patients receiving at least one dose of medication (intent-to-treat). Patients who did not complete the treatment regimen were classified as nonresponders. In order to investigate the risk factors, analyses of associations and logistic regression were performed in patients who had completed at least 24 weeks of treatment.

The histological analysis was performed by a pathologist, and the fibrosis was classified using the Brazilian Society of Pathology system [9]: F0 = no fibrosis; F1 = portal fibrosis without septa; F2 = fibrous expansion with rare septa; F3 = numerous septa with the formation of some nodules but with partial preservation of the hepatic architecture; F4 = cirrhosis.

The necro-inflammatory activity was graded in the various regions as follows: portal $(0=$ few lymphocytes, $1=$ discrete increase in the number of lymphocytes, $2=$ moderate increase in the number of lymphocytes, $3=$ marked increase in the number of lymphocytes, and $4=$ highly pronounced increase in the number of lymphocytes); periportal $(0=$ no interface lesions; $1=$ extravasation ('spillover') of lymphocytes into the interface, $2=$ discrete piecemeal necrosis, $3=$ moderate piecemeal necrosis, and $4=$ extensive piecemeal necrosis throughout many of the portal spaces $)$; lobular $(0=$ normal hepatocytes; 1 = rare foci of necrosis and rare lymphohistiocytic infiltrate (LHI), 2 = focal necrosis of hepatocytes and LHI at numerous sites, $3=$ focal necrosis of hepatocytes and LHI at numerous sites, accompanied by limited areas of confluent necrosis, and $4=$ focal necrosis of hepatocytes and LHI at numerous sites, with extensive or multiple areas of confluent necrosis) [9]. We subcategorized the necro-inflammatory activity as follows: portal inflammatory infiltrate activity $(\mathrm{A}=$ discrete number of lymphocytes: grades 0 to 1 and $\mathrm{B}=$ moderate to marked increase in the number of lymphocytes: grades 2 to 4$)$; periportal activity $(\mathrm{A}=$ no interface lesions or spillover of lymphocytes into the interface: grades 0 to 1 , and $\mathrm{B}=$ discrete to marked piecemeal necrosis: grades 2 to 4 ).

The levels of HCV RNA were determined using PCR (Amplicor; Roche Diagnostics, Hoffmann-La Roche Ltd, Basel, Switzerland). The HCV genotype was determined using reverse hybridization (INNO-LiPA HCV II; Innogenetics, NV, Ghent, Belgium).

All participating patients gave written informed consent, and the study design was approved by the Ethics in Research Committee of the Botucatu School of Medicine.

\section{$\underline{\text { Statistical Analysis }}$}

The nominal variables are presented in absolute and relative frequencies in the tables. For the continuous variables, the descriptive statistics are presented as mean and standard deviation, with minimum and maximum intervals.

The analyses of associations between and among the SVR, nonresponder and relapser groups were performed using the chi-square and Fisher's exact tests. The analyzed variables were classified as follows: body mass index (BMI, $<$ or $\geq 25$ $\mathrm{Kg} / \mathrm{m}^{2}$ ), age ( $<$ or $\geq 40$ years); fibrosis (stage $\leq 2$ or stages $3 / 4$ ); portal inflammatory activity (portal inflammatory infiltrate: A or B); periportal inflammatory activity (piecemeal necrosis: A or B); alanine aminotransferase [ALT of $\leq 1,1-3$, or $>3 \times$ the upper limit of normality (ULN)]; aspartate aminotransferase (AST, $\leq 1,1-3$, or $>3 \times$ the ULN); gamma-glutamyl transpeptidase (GGT, $\leq 1,1-3$, or $>3 \times$ the $\mathrm{ULN}$ ); race (White, non-White, or Asian); and platelet count $\left(<\right.$ or $\left.\geq 150,000 / \mathrm{mm}^{3}\right)$.

Polytomous logistic regression was used to determine the $\mathrm{p}$ values and odds ratios (ORs), together with $95 \%$ confidence intervals (95\% CIs), for the effect that the prognostic values have on the transition from responder to relapser. The independent variables included were as follows: age; fibrosis; portal and periportal inflammatory activity; BMI; ALT; AST; GGT; gender; weight differences between the initiation of treatment and week 12 (D weight); and HCV RNA levels at weeks 12 and 24 of treatment.

The level of significance adopted was $5 \%$. The tests were performed using the SAS System for Windows, version 6.12 (SAS Institute Inc., Cary, NC, USA).

\section{Results}

Of the 84 patients, 26 were excluded: seven for having been submitted to treatment with pegylated interferon alfa $2 \mathrm{a}$; 18 for having been submitted to retreatment; and one for being 
co-infected with hepatitis B virus. Therefore, 58 intent-to-treat patients were examined.

Table 1 shows the pre-treatment characteristics of the 58 patients included in the study. Most $(81 \%)$ were men, and patients with grades F3 or F4 of fibrosis (numerous septa or numerous septa accompanied by cirrhosis) represented $26 \%$ of the sample.

Table 1. Demographic and biochemical profile of the 58 patients included in the study

\begin{tabular}{ll}
\hline Variable & \\
\hline Age $(\text { years })^{*}$ & $40.5 \pm 10.8(21-67)$ \\
Weight $(\mathrm{Kg})^{*}$ & $72.2 \pm 15.4(34.7-113)$ \\
BMI $\left(\mathrm{Kg} / \mathrm{m}^{2}\right)^{*}$ & $25.1 \pm 3.8(17.5-34.7)$ \\
AST $(\#$ of $\times \mathrm{ULN}) *$ & $1.4 \pm 2.0(0.2-12.8)$ \\
ALT $(\# \text { of } \times \mathrm{ULN})^{*}$ & $1.8 \pm 2.7(0.2-17)$ \\
GGT $(\#$ of $\times \mathrm{ULN}) *$ & $1.4 \pm 1.5(0.2-6.7)$ \\
Gender, M/F $(\%$ male) & $47 / 11(81 \%)$ \\
Race $(\mathrm{White} /$ non-White/Asian $)$ & $78 / 20 / 2(\%)$ \\
\hline
\end{tabular}

* Mean \pm SD (range). BMI: body mass index; AST: aspartate aminotransferase; ULN: upper limit of normality; ALT: alanine aminotransferase; GGT: gamma-glutamyl transpeptidase.

Sustained virological responders versus nonresponders versus relapsers

In intent-to-treat patients, the virological response was $69 \%$ (39/58) at the end of treatment, and an SVR was achieved in $52 \%(30 / 58)$ (Figure 1).

Figure 1. Percentages of patients in the intent-to-treat population $(\mathrm{n}=58)$ presenting end-of-treatment virological response (ETVR) and sustained virological response (SVR).

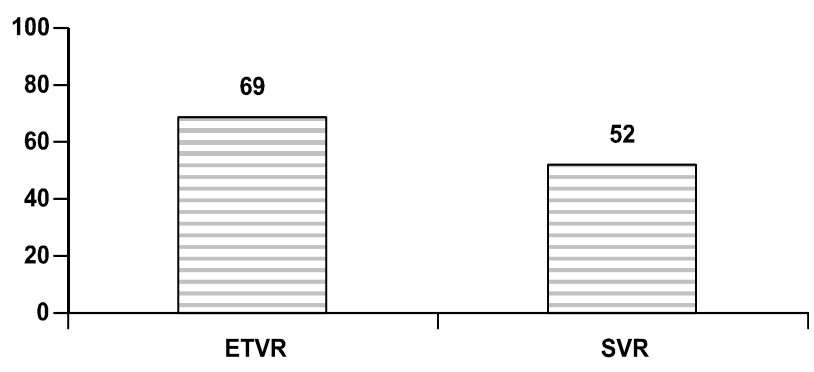

Of the 58 patients, five did not complete the 24 weeks of treatment and were excluded from the analysis of the factors associated with being a sustained virological responder, a nonresponder, or a relapser.

In the 'on-treatment' analysis, we found that 30 patients $(57 \%)$ were sustained virological responders, 14 (26\%) were nonresponders, and nine (17\%) were relapsers (Figure 2).

We observed a significant association between SVR and fibrosis $\leq$ F2 $(\mathrm{p}=0.04)$, as well as between SVR and
Figure 2. Percentages of patients in the on-treatment population $(n=53)$ presenting end-of-treatment virological response (ETVR), sustained virological response (SVR), no response (NR), and relapse (REL).

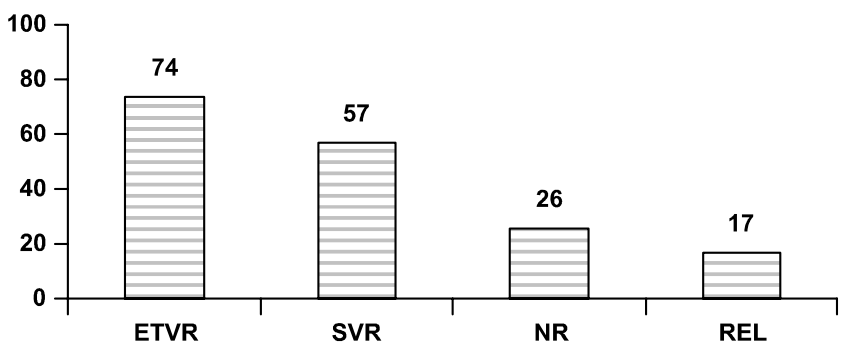

undetectable levels of HCV RNA at weeks 12 and 24 of treatment $(\mathrm{p}<0.0001)$. We observed no statistical differences among the three groups in terms of BMI, age, ALT, AST, GGT, platelet count, gender, or race (Table 2).

Table 2. Distribution of the frequencies of the variables studied according to the type of treatment response

\begin{tabular}{|c|c|c|}
\hline Variable & $\begin{array}{c}\text { SVR } v s \text { NR } v s \text { REL } \\
(\%)\end{array}$ & p value \\
\hline Fibrosis (stage F3 or F4) & 10 vs 43 vs 22 & $0.04 *$ \\
\hline $\operatorname{BMI}\left(>25 \mathrm{Kg} / \mathrm{m}^{2}\right)$ & 43 vs 57 vs 67 & 0.4 \\
\hline Age ( $>40$ years) & 40 vs 50 vs 78 & 0.1 \\
\hline $\operatorname{ALT}(>3 \times$ the $\mathrm{ULN})$ & $26 v s 21$ vs 22 & 0.8 \\
\hline $\operatorname{AST}(>3 \times$ the ULN) & $7 v s 21$ vs 11 & 0.6 \\
\hline GGT ( $>3 \times$ the ULN) & $7 v s 21$ vs 22 & 0.5 \\
\hline Platelet count $\left(>150 \times 10^{3}\right)$ & 90 vs 71 vs 78 & 0.2 \\
\hline
\end{tabular}

SVR: sustained virological responder group; NR: nonresponder group; REL: relapser group; F3/F4: fibrosis units; BMI: body mass index; ALT: alanine aminotransferase; ULN (upper limit of normality); AST: aspartate aminotransferase; GGT (gamma-glutamyl transpeptidase).

In analyzing the sustained virological responder and relapser groups independently, we observed that being over the age of 40 was significantly associated with being a relapser $(\mathrm{p}=0.04)$. Among the relapsers, $78 \%$ were older than 40 , compared with only $40 \%$ of the sustained virological responders. As can be seen in Table 3, no significant differences between the sustained virological responder and relapser groups were found in terms of the remaining variables (fibrosis, BMI, portal inflammatory infiltrate, periportal inflammatory infiltrate, race, ALT, AST, and GGT).

An additional analysis was carried out in order to identify any possible associations between the sustained virological responder and nonresponder groups. We found that being a nonresponder was significantly associated with severe (F3 or F4) fibrosis $(\mathrm{p}=0.01)$, with moderate to marked portal inflammatory infiltrate (grade $\mathrm{B})(\mathrm{p}=0.01)$, and with piecemeal necrosis (grade $\mathrm{B})(\mathrm{p}=0.02)$. As also shown in Table 3 , no 
Table 3. Distribution of the frequencies of the variables studied according to the type of treatment response: 2-by-2 comparisons

\begin{tabular}{|c|c|c|c|}
\hline Variable & SVR vs NR vs REL (\%) & SVR vs NR p value & SVR $v$ s REL $p$ value \\
\hline Fibrosis (stage F3 or F4) & $10 v s 43$ vs 22 & $0.01 *$ & 0.33 \\
\hline $\mathrm{BMI}\left(>25 \mathrm{Kg} / \mathrm{m}^{2}\right)$ & 43 vs 57 vs 67 & 0.39 & 0.21 \\
\hline Age $(>40$ years $)$ & 40 vs 50 vs 78 & 0.53 & $0.04 *$ \\
\hline $\operatorname{ALT}(>3 \times \mathrm{ULN})$ & 26 vs 21 vs 22 & 0.80 & 0.59 \\
\hline $\operatorname{AST}(>3 \times \mathrm{ULN})$ & $7 v s 21$ vs 11 & 0.28 & 0.89 \\
\hline GGT $(>3 \times$ ULN $)$ & $7 v s 21 v s 22$ & 0.31 & 0.38 \\
\hline Platelet count $\left(>150 \times 10^{3}\right)$ & 90 vs 71 vs 78 & 0.11 & 0.33 \\
\hline Grade B Portal inflammatory activity & 57 vs 93 vs 67 & $0.01 *$ & 0.50 \\
\hline Grade B Periportal inflammatory activity & $50 v s 85$ vs 55 & $0.02 *$ & 0.76 \\
\hline
\end{tabular}

SVR: sustained virological responder group; NR: nonresponder group; REL: relapser group; F3/F4: fibrosis units; BMI: body mass index; ALT: alanine aminotransferase; ULN (upper limit of normality); AST: aspartate aminotransferase; GGT (gamma-glutamyl transpeptidase); Grade B portal inflammatory activity: moderate to marked number of portal lymphocytes; Grade B periportal inflammatory activity: discrete to accentuated piecemeal necrosis.

statistical differences were found between the sustained virological responder and nonresponder groups in terms of the remaining variables (age, BMI, race, ALT, AST, and GGT).

\section{Predictors of Relapse}

In the polytomous logistic regression analysis, we attempted to determine which variables might explain the transition from responder to relapser. We included the following variables in the model: age; fibrosis; BMI; ALT; AST; GGT; gender; weight differences between the initiation of treatment and week 12 ( $\Delta$ weight); and HCV RNA levels at weeks 12 and 24 of treatment. None of the studied variables, except HCV RNA levels at weeks 12 and 24 of treatment, were capable of explaining the relapse response

Figure 3 shows the evolution of the mean weight over the course of the treatment (at weeks 12 and 48) compared to the baseline weight. We observed that mean weight had dropped by $5 \%$ at week 12 of treatment and by $10 \%$ at the end of treatment.

The polytomous logistic regression analysis revealed no differences between the sustained virological responder and

Figure 3 Average patient body weights and standard deviations at three time points during the treatment period: baseline; week 12 of treatment (tw12); and week 48 of treatment (tw48).

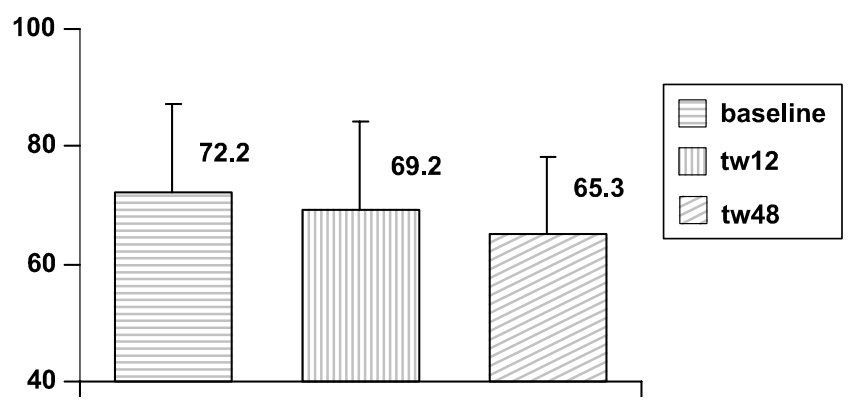

relapser groups in the independent variables analyzed $(\mathrm{p}=$ 0.1). In the results of the tests employed to detect HCV RNA at weeks 12 and 24 of treatment, it was not possible to differentiate between sustained virological responders and relapsers (Table 4). All sustained virological responders presented undetectable HCV RNA at weeks 12 and 24 of treatment, compared with $78 \%$ and $67 \%$ of the relapsers at weeks 12 and 24, respectively (Table 5).

In this regression analysis, we found a significant difference between relapsers and nonresponders when we compared them in terms of the independent variable HCV RNA level at week 12 of treatment $(\mathrm{p}=0.008)$. Patients with detectable HCV RNA levels at week 12 of treatment presented a 4.8-times greater chance of being nonresponders (Table 4). When we substituted detectable HCV RNA levels at week 24 of treatment for detectable HCV RNA levels at week 12 of treatment in the logistic regression analysis, the OR was 8.6 (95\% CI: 2.1-33.3; $\mathrm{p}=0.001)$.

Table 5 shows that HCV RNA was undetectable in $36 \%$ of the nonresponders at week 12 of treatment, compared with $21 \%$ at week 24 . In addition, all relapsers presented detectable HCV RNA levels by post-treatment week 12 (early relapse of the infection).

\section{Discussion}

Historically, chronic HCV infection has been a difficult-totreat disease. However, in recent years, great progress has been made in terms of achieving an SVR, especially in patients infected with HCV genotypes 2 or 3. Despite this advance, less satisfactory results have been achieved in certain groups of patients, namely those infected with HCV genotype 1 .

The first studies involved the administration of conventional interferon and were followed by those employing the interferon-ribavirin combination and extending the treatment period from 24 to 48 weeks, resulting in a significant (300\%) increase in SVR rates (from 10 to 36\%) [10,11]. However, an SVR is still achieved in only one-third of the population submitted to treatment. 
Table 4. Risk factors associated with relapse - polytomous logistic regression

\begin{tabular}{lccc}
\hline Parameter & Chi-square p value & 95\% confidence interval & Odds ratio \\
\hline Intercept 0 (REL $v s$ SVR) & 0.110 & \\
Intercept 1 (REL $v$ NR) & 0.008 & $1.182-20.0$ & 4.8 \\
Detectable HCV RNA at tw12 & 0.028 & $0.707-7.46$ & \\
Age & 0.170 & $0.730-1.13$ \\
$\Delta$ weight (baseline - tw12) & 0.166 & $0.670-9.61$ \\
Fibrosis & 0.407 & \\
\hline
\end{tabular}

SVR: sustained virological responder group; NR: nonresponder group; REL: relapser group; tw12 (week 12 of treatment); D weight (baseline weight minus weight at tw12).

Table 5. Undetectable levels of HCV RNA, by week

\begin{tabular}{|c|c|c|c|c|c|}
\hline \multirow[b]{2}{*}{ Subgroup } & \multicolumn{3}{|c|}{ Treatment period } & \multicolumn{2}{|c|}{ Post-treatment period } \\
\hline & Week 12 N (\%) & Week 24 N (\%) & Week 48 N (\%) & Week 12 N (\%) & Week 24 N (\%) \\
\hline $\operatorname{SVR}(n=30)$ & $30(100 \%)$ & $30(100 \%)$ & $30(100 \%)$ & $30(100 \%)$ & $30(100 \%)$ \\
\hline $\mathrm{NR} \quad(n=14)$ & $5(36 \%)$ & $3(21 \%)$ & $0(0 \%)$ & $0(0 \%)$ & $0(0 \%)$ \\
\hline REL (n=9) & $7(78 \%)$ & $6(67 \%)$ & $9(100 \%)$ & $0(0 \%)$ & $0(0 \%)$ \\
\hline
\end{tabular}

SVR: sustained virological responder; NR: nonresponder; REL: relapser.

More recently, two separate studies demonstrated that, in patients infected with HCV genotype 1, the pegylated interferonribavirin combination administered for a period of 48 weeks resulted in substantially higher SVR rates than did the combination of standard interferon and ribavirin administered for the same length of time (42\% vs 33\% and $46 \%$ vs 36\%, respectively, in both studies) [3,4]. Among the patients infected with genotype 1 , various factors have been associated with the absence of an SVR. Such factors include, but are not limited to, the following: high BMI or weight $>75 \mathrm{Kg}$; fibrotic foci and cirrhosis; being younger than 40 years of age; high viral load before treatment; viral clearance by week 12 of treatment; obesity; hepatic steatosis; and noncompliance with treatment.

Some patients achieving an SVR by the end of treatment present detectable HCV RNA levels during the follow-up period (recurrence of the infection). Studies have shown relapse rates of $13 \%$ to $18 \%$ among patients treated with the pegylated interferon-ribavirin combination $[3,4]$. If identified early, these patients could be submitted to longer periods of treatment, which might produce an increase in the SVR rate. Buti et al. [12] obtained higher SVR rates in patients whose treatment was extended to 72 weeks. However, the study design was to extend the treatment for patients in whom HCV RNA was detectable at week 12 of treatment and undetectable at week 24 (slow responders). The increase in SVR found in the study might have been achieved by decreasing the relapse rate. Therefore, it would be interesting if we could determine which patients will present detectable HCV RNA after the end of treatment. Such knowledge would inform the decision of whether or not to extend the treatment.

The present study was carried out in an outpatient clinic of a university hospital and involved 58 consecutive patients meeting the criteria established by the Brazilian Ministry of
Health for treatment of chronic $\mathrm{HCV}$ infection. It is, therefore, a sample characterizing a real-world population. At the time of this study, the guidelines established by the State of São Paulo Department of Health stated that patients with HCV genotype 1 infection should receive pegylated interferon alfa $2 \mathrm{~b}$, which was doled out to the various clinics in a rationing effort. The SVR rate found in the present study (52\% in intentto-treat patients) is similar to or higher than that found in large published trials [3-5]. We believe that this favorable SVR rate can be explained by the higher patient adherence to the treatment, since this is known to be an extremely important factor for achieving an SVR. We attribute the high rate of compliance to the multidisciplinary approach taken to the treatment of patients at the facility.

Of the major published studies of patients receiving treatment with the pegylated interferon-ribavirin combination, none have made a comparative analysis of the nonresponders and the relapsers as separate groups [3-5]. Therefore, we attempted to evaluate potential differences between and among sustained virological responders, nonresponders, and relapsers. We also had the objective of identifying factors predictive of the transition from responder to relapser.

Interestingly, when we evaluated the statistical differences between the sustained virological responder and relapser groups, we found that only the 'being more than 40 years of age' variable was significantly associated with being a relapser. Advanced fibrosis was not associated with being a relapser. The results of various studies involving the administration of pegylated interferon, with or without ribavirin, have shown that being younger than 40 years of age is predictive of achieving an SVR, regardless of the type of pegylated interferon employed $[3,4,14,15]$. Therefore, whereas previous analyses predicted only SVR, one aspect of our study was 
the prediction of relapse. The association found between being older than 40 and being a relapser was not found in relation to being a sustained virological responder or being a nonresponder. However, our study was limited by the small sample size, and our results should therefore be validated in further studies involving larger patient samples.

The analysis of the sustained virological responder and nonresponder groups showed that being a nonresponder is associated with severe fibrosis, marked portal inflammatory infiltrate, and piecemeal necrosis (Table 3). The presence of severe fibrosis is recognized as a factor that is independent for a decrease in SVR $[3,16]$. It is of note that we did not observe significant differences between the sustained virological responder and relapser groups in terms of the fibrosis variable. Therefore, relapse occurred in the absence of severe fibrosis.

We did not find SVR to be associated with BMI, aminotransferase activity levels, GGT levels, race, or gender. In a previous study involving administration of pegylated interferon alfa $2 b$, lower body weight was not found to be associated with SVR [3]. However, such an association was found in a study involving pegylated interferon alfa $2 \mathrm{a}$ [4]. It has been reported that African-Americans have a lower chance of achieving an SVR than do other ethnic groups [17]. We did not observe any ethnicity-based differences in our study. However, ethnicity can not be given much importance in Brazil, where there has been extensive miscegenation.

The polytomous logistic regression analysis did not identify any independent factors associated with the relapser group in comparison with the sustained virological responder group. Even HVC RNA being undetectable at week 12 of treatment did not differ between the sustained virological responder and relapser groups $(100 \%$ in sustained virological responders and $78 \%$ in relapsers; Table 4). Therefore, HCV RNA clearance by week 12 of treatment was not predictive of being a relapser or of being a sustained virological responder (Table 5). The regression analysis revealed differences between the relapser and nonresponder groups in terms of HCV RNA levels at weeks 12 and 24 of treatment. Detectable level of HCV RNA at weeks 12 and 24 of treatment was predictive of being a nonresponder, increasing the chance by 4.8 and 8.6 times, respectively, when compared to the relapsers.

In all relapsers, HCV RNA levels again became detectable by post-treatment week 12 (Table 4 ). Therefore, we can suggest that treatment-naïve patients infected with $\mathrm{HCV}$ genotype 1 and receiving pegylated interferon alfa $2 \mathrm{~b}$ combined with ribavirin should not be classified as presenting an SVR unless HCV RNA remains undetectable until three months after the end of treatment. Zeuzem et al. [18] evaluated 1,441 patients infected with HCV genotype 1 or with another genotype and submitted to treatment with standard or pegylated interferon. The authors observed that the recurrence of the infection occurred by post-treatment week 12 in $98 \%$ of the cases. Analyses of baseline characteristics have failed to identify parameters associated with early relapse.
We conclude that, in our study sample (treatment-naïve patients infected with genotype 1 and treated with the pegylated interferon alfa 2b-ribavirin combination), an SVR was achieved in $52 \%$ of the intent-to-treat patients. It is likely that this occurred due to the high rate of patient adherence to treatment resulting from the multidisciplinary attention provided at our facility. Relapser and nonresponder groups differed from each other when compared to the sustained virological responder group. Recurrence of the infection occurred in $17 \%$ of the cases (on-treatment) and was associated with being over the age of 40 . However, it was not associated with other factors, such as severe fibrosis. From the results of the HCV RNA tests performed at weeks 12 and 24 of treatment, it was not possible to differentiate between sustained virological responders and relapsers, although those values were found to be independent predictors of being a nonresponder. We suggest that further studies, involving larger patient samples, should be carried out in order to validate our findings.

\section{References}

1. World Health Organization (WHO). Hepatitis C - global prevalence. Weekly Epidemiological Record 1997;72:341-4.

2. National Institutes of Health Consensus Development Conference Panel statement: management of Hepatitis C. Hepatology 1997;26(suppl 1):2-10.

3. Manns M.P., McHutchison J.G., Gordon S.C., et al. Peginterferon alfa-2b plus ribavirin compared with interferon alfa $2-b$ plus ribavirin for initial treatment of chronic hepatitis $\mathrm{C}$ : a randomised trial. Lancet 2001;358:958-65.

4. Fried M.W., Shiffman M.L., Reddy K.R., et al. Peginterferon alfa2a plus ribavirin for chronic hepatitis $\mathrm{C}$ virus infection. N Engl J Med 2002;347:975-82.

5. Hadziyannis S.J., Sette H.J., Morgan T.R., et al.; PEGASYS International Study Group. Peginterferon alfa-2a and ribavirin combination therapy in chronic hepatitis $\mathrm{C}$ : a randomized study of treatment duration and ribavirin dose. Ann Intern Med 2004; $140: 346-55$.

6. Poynard T., Marcellin P., Lee S.S., et al. Randomised trial of interferon alfa-2b plus ribavirin for 48 weeks or for 24 weeks versus interferon alfa- $2 \mathrm{~b}$ plus placebo for 48 weeks for treatment of chronic infection with hepatitis $\mathrm{C}$ virus. Lancet 1998; $352: 1426-32$.

7. Marcellin P., Boyer N., Gevais A., et al. Long-term histologic improvement and loss of detectable intrahepatic HCV RNA in patients with chronic hepatitis $\mathrm{C}$ and sustained response to interferon-alpha therapy. Ann Intern Med 1997;127:875-81.

8. Poynard T., McHutchinson J., Manns M., et al. The impact pegylated interferon alpha 2-b and ribavirin on liver fibrosis in patients with chronic hepatitis C. Gastroenterology 2002; 122 : $1303-13$.

9. Gayotto \& Comitê da Sociedade Brasileira de Patologia. Visão histórica e consenso nacional sobre a classificação das hepatites crônicas. Gastroenterologia e Endoscopia Digestiva, 2000;19(3):137-40.

10. McHutchison J.G., Gordon S.C., Schiff E.R., et al.; Hepatitis Interventional Therapy Group. Interferon alfa 2-b alone or in combination with ribavirin as initial treatment for chronic hepatitis C. N Engl J Med 1998;339:1485-92.

11. Poynard T., Marcellin P., Lee S.S., et al. Hepatitis Interventional Therapy Group. Randomized trial of interferon $\alpha 2 b$ plus ribavirin for 48 weeks or for 24 weeks versus interferon $\alpha 2 \mathrm{~b}$ plus placebo for 48 weeks for treatment of chronic infection with hepatitis $\mathrm{C}$ virus. Lancet 1998;352:1426-32. 
12. Buti M., Valdes A., Sanches-Avila F., et al. Extending combination therapy with peginterferon alfa 2-b plus ribavirin for genotype 1 chronic hepatitis $C$ late responders : a report of 9 cases. Hepatology 2003;37:1226-7.

13. McHutchinson J.G., Manns M., Patel K., et al. Adherence to combination therapy enhances sustained response in genotype 1 infected patients with chronic hepatitis C. Gastroenterology 2002;123:1061-9.

14. Zeuzem S., Feinman S.V., Rasenack J., et al. Peginterferon alfa 2$\mathrm{a}$ in patients with chronic hepatitis C. N Engl J Med 2000;343:1666-72.

15. Foster G.R., Fried M.W., Hadziyannis S.J., Chaneac M. Treatment of chronic hepatitis $\mathrm{C}$ with peginterferon alfa 2 -a and ribavirin: patient age has a marked influence on the individual estimated probability of achieving a sustained virological response [abstract]. Hepatology 2003;38(Suppl1):246A.

16. Zeuzem $S$. Heterogeneous virologic response rates to interferonbased therapy in patients with chronic hepatitis $\mathrm{C}$ : who responds less well? Ann Intern Med 2004;140:370-81

17. Howell C., Jeffers L., Hoofnagle J.H. Hepatitis C in African Americans: summary of a workshop. Gastroenterology 2000; $119: 1385-96$

18. Zeuzem S, Heathcote JE, Shiffman ML et al. Twelve weeks of follow-up is sufficient for the determination of sustained virologic response in patients treated with interferon a for chronic hepatitis C. J Hepatol 2003;39:106-11. 